\title{
Subjective time perception is affected by different durations of exposure to abstract paintings that represent human movement
}

\author{
Francisco Carlos Nather ${ }^{1}$, Paola Alarcon Monteiro Fernandes ${ }^{2}$, and José Lino Oliveira Bueno ${ }^{2}$ \\ 1- Universidade Federal do ABC, São Bernardo do Campo, SP, Brazil \\ 2- Universidade de São Paulo, Ribeirão Preto, SP, Brazil
}

\begin{abstract}
Time perception can be affected by real emotional pictures of people that evoke different levels of arousal. Figurative artwork images of body postures that imply movement with different intensities and evoke different levels of arousal can modulate the perception of time. The present study investigated whether abstract paintings that represent motion in different ways affect the perception of time when subjects are exposed to the paintings for different durations. Undergraduate students observed 20 abstract paintings from different artistic schools (i.e., cubism, constructivism, expressionism, and futurism). They observed for $3 \mathrm{~s}$ each painting and estimated the time of exposure (reproduction method). After the time estimations, the subjects completed different semantic Movement, Arousal, Complexity, and Recognition scales to obtain information about how the painting compositions were perceived. Time distortions were observed for only two cubist paintings that represented human forms, which were related to both evoked arousal and implied movement (Experiment 1). Experiment 2 further verified whether these time distortions were related to implied movement perception or arousal. Different groups of participants were exposed for 3 and $9 \mathrm{~s}$ to only four cubist paintings that represented human forms. These time exposures ( 3 and $9 \mathrm{~s}$ ) were used because the arousal-evoking effects may be transient for exposure times that are longer than 2-3 s. The data analysis revealed overestimation of time for the cubist painting that had greater arousal and movement scores only when the subjects were exposed for $9 \mathrm{~s}$, showing that implied movement in abstract human figures is more effective than images with emotional content. We discuss the effect of durations of exposure to pictorial characteristics of artwork on time perception, emphasizing aspects of the visual perception of human forms in cubist paintings and arousal effects in an aesthetic episode. Keywords: time perception, static images, implied movement, cubism, arousal.
\end{abstract}

Received 22 April 2014; received in revised form 04 August 2014; accepted 15 August 2014. Available online 25 November 2014.

\section{Introduction}

Impressionist artwork by Edgar Degas that represents ballerina movements modulates subjective time perception. Ballet steps with lower movement scores were judged to have a shorter duration than those with intermediate scores, and ballet postures with intermediate scores were judged to have a shorter duration than postures with the highest scores, such as arabesque and attitude postures (Nather \& Bueno, 2012). According to these authors, arabesque and attitude postures are more asymmetric than other ballet

Francisco Carlos Nather, Universidade Federal do ABC, Center for Mathematics, Computation and Cognition. José Lino Oliveira Bueno, University of São Paulo, Department of Psychology. Paola Alarcon Monteiro Fernandes, University of São Paulo, Department of Biology. Correspondence regarding this article should be directed to: Francisco Carlos Nather, Centro de Matemática, Computação e Cognição (CMCC) Universidade Federal do ABC, Rua Arcturus, 03, São Bernardo do Campo, SP 09606-070, Brazil. Phone: +55 1123206270 Fax: +551123206120. E-mail: f.nather@hotmail.com/f.nather@ ufabc.edu.br postures because of the relationship among the positions of the body parts, allowing the observer to surmise the represented movements (implied movement).

Implied motion in abstract and figurative artworks has been studied under different aspects of visual perception that involves neuronal substrates and subjective time (Braddick, 1995; Freedberg \& Gallese, 2007; Kourtzi, 2004; Martinez-Conde \& Macknik, 2010; Nather \& Bueno, 2006, 2012). According to Cutting (2002), painters use at least five criteria to represent motion, such as relationships among positions of body parts (broken symmetry), blurring the background of the scene (photographic blur), and superimposing different moments of a moving object in the same painting (stroboscopic images). For example, in the abstract cubist painting Nude Descending a Staircase by Marcel Duchamp, the human figure is repeatedly overlapped to show all of the human body movements while descending stairs. Similarly, the futurist painting Dynamism of a Dog on a Leash by Giacomo Balla shows the movements made by a dog using stroboscopic images and blurs the scene background. 
Kim and Blake (2007) studied 24 paintings from the early 20th century and found that subjects were able to distinguish movement intensities in different artworks. Paintings that portrayed motion were rated with higher scores than paintings in which motion was not explicitly intended by the artists. They found greater activity for the motion-sensitive MT+ encephalic brain area in Nude Descending a Staircase and Dynamism of a Dog on a Leash (which imply more movement) compared with abstract expressionist paintings (which imply less movement) in observers who had prior viewing experience with these artworks. In these experiments, the authors also used expressionist paintings by Paul Klee and Piet Mondrian that are basically composed of geometric forms and lines. Thakral, Moo, and Slotnick (2012) evaluated the magnitude of activity in the MT+ sensory motion processing region and prefrontal cortex while subjects viewed 20 other paintings (i.e., portraits and landscapes by Van Gogh). Based on each subject's motion experience ranking, the authors showed a significant positive correlation between the intensity of movement associated with viewing each painting and the magnitude of activity of the MT + area. Furthermore, the paintings that were classified by the participants as pleasant were associated with greater activity of the MT+ area compared with paintings that were classified as unpleasant. These results suggested that the activity of this brain area is associated with the experience of both movement and aesthetic pleasure.

The effects of aesthetic episodes on time perception has revealed that it varies directly with the pictorial processing of the artwork's characteristics. They determined how much time an observer spends in cognitive processing to understand a piece of art (Cupchick, 1976; Cupchick \& Gebotys, 1988). Using paintings by Claude Monet, Edgar Degas, and Édouard Manet, to which subjects were exposed for 18, 36, and $72 \mathrm{~s}$, Cupchick and Gebotys (1988) found that pleasure judgments by the subjects varied according to the complexity of these artworks and the duration of exposure. However, this study did not focus on specific properties of the paintings' themes, such as the effects of movement perception on subjective time.

Freedberg and Gallese (2007) proposed that an element of aesthetic episodes consists of the activation of embodiment mechanisms that encompass the simulation of actions, emotions, and corporeal sensations by motion-sensitive cortical brain areas, such as mirror neurons. Edgar Degas is widely known for his artistic productions that are dedicated to the representation of movement (Growe, 2001). His realistic sculptures of ballerina body postures likely affect the $\mathrm{MT}+$ area because cortical brain areas are differentially activated by real pictures of people's postures while they perform different movements. For example, the observation of an athlete who is performing an action causes more activation of the MT/MST than this same athlete who is in a static posture (Kourtzi \& Kanwisher, 2000; Proverbio, Riva, \& Zani, 2009). Embodiment mechanisms have been associated with time distortions in previous studies that used body postures of dancers and an exposure time of $36 \mathrm{~s}$ (Nather \& Bueno, 2011). Nather, Bueno, Bigand, and Droit-Volet (2011) analyzed several aspects of motion perception, including arousal, valence, and familiarity with Degas' ballerina images, and evaluated the impact of empathy and embodiment mechanisms in an aesthetic episode. They showed that the duration of exposure was judged to be longer for ballerina images that implied more movement compared with ballerina images that implied less movement. However, the magnitude of the time distortions was greater for the shorter durations of exposure (0.4-1.6 s) than for the longer durations (2-8 s). Interestingly, the authors found a positive relationship between the intensity of movement and arousal. Body postures that implied more movement were judged as more arousing than body postures that implied less movement. They explained the difference in time distortions between the shorter (milliseconds) and longer (a few seconds) durations of stimulus exposure by relating it to emotional decay in which the high-arousal effect was transient and limited to relatively brief durations that were shorter than 2-3 s (Angrili, Cherubini, Pavese, \& Manfredini, 1997). Angrili et al. hypothesized that the effects of high-arousal real human pictures were mostly emotional until the first $2 \mathrm{~s}$ of exposure, which then decreased after $4 \mathrm{~s}$. Thus, the emotional content of the images would be given priority during the early phase of time processing, and attention becomes more effective when the exposure duration is approximately $6 \mathrm{~s}$ (short interval).

Arousal is a fundamental mechanism that modulates the effect of emotion, likely in multiple stages of time processing when a person observes emotional human pictures (Smith, McIver, Di Nella, \& Crease, 2011). The effects of aesthetic episodes on time perception has revealed that the perception of the duration of exposure also varies according to the artwork's properties (Berlyne, 1974). Thus, understanding the effects of arousal and implied motion in non-figurative artworks could elucidate the processes that underlie embodiment mechanisms that impact subjective time because "...the feeling of physical involvement in artworks not only provoked a sense of imitating the motion seen or implied in the work, but also enhanced the spectator's emotional responses to it" (Freedberg \& Gallese, 2007, p. 198).

The objective of the present study was to investigate the effects of arousal and the perception of movement in abstract paintings on time perception by testing short and long durations of exposure to artwork. We tested whether time perception is differentially affected by different durations of exposure to artwork, the perception of implied movement, and the evocation of arousal. To evaluate the effects of implied movement and arousal on time perception, paintings with different themes that were produced during different artistic movements were used in two experiments, in which the paintings were presented for $3 \mathrm{~s}$ (Experiment 1) and 3 and $9 \mathrm{~s}$ 
(Experiment 2) in different groups of participants. In Experiment 1, 20 paintings that represented cubism, constructivism, expressionism, and futurism were used. They were selected because their different motion representation characteristics (Cutting, 2002) have not been tested in previous studies of subjective time (e.g., stroboscopic images, pictures with photographic blur, and action-movement paintings).

Four cubist paintings were used in Experiment 2. They consisted of abstract human forms. These cubist paintings were selected because at least two of them were perceived as figurative human forms when subjects viewed them for more than $7.5 \mathrm{~s}$ (Hekkert $\&$ van Wieringen, 1990). Therefore, two durations of exposure ( 3 and $9 \mathrm{~s}$ ) were used in Experiment 2 because these paintings were not easily recognized as human forms with a short duration of exposure (3 s). Furthermore, high-arousal effects can be transient with exposure times greater than 3-4 s in which time perception is mediated by different cognitive processes with 6 s exposure (Angrilli et al., 1997; Elbert, Ulrich, Rockstroh, \& Lutzenberger, 1991). Thus, exposure to the same paintings for 3 and $9 \mathrm{~s}$ was used to test the specific effects of implied movement in abstract human forms and the effects of emotional arousal on time perception. If the effects of arousal decrease briefly at $6 \mathrm{~s}$, then the time estimations for $9 \mathrm{~s}$ exposure are more related to implied movement than to an arousal effect.

The present study also sought to clarify aspects of visual motion perception that involves an aesthetic episode in which different kinds of implied movement are the most conspicuous characteristics of the abstract compositions. Notably, implied movements were not evaluated in previous studies. Previous studies of subjective time used figurative artwork that was realistically distinguished as women who were dancing with body postures that were associated with different ballet choreographies, which affected time perception according to visual motion perception. The implicit visual information of these static images of different body postures revealed that movement perception modulates time perception according to increases in motion intensity (Nather \& Bueno, 2011). Therefore, the present study also evaluated whether abstract human forms in abstract paintings cause similar effects.

\section{EXPERIMENT 1}

\section{Methods}

\section{Participants}

Fifteen undergraduate students (eight men; mean age $=20.26$ years, $\mathrm{SD}=2.12$ ) from the University of São Paulo, Ribeirão Preto, Brazil were randomly invited to participate in the study. They were untrained in visual arts and had normal or corrected-to-normal vision. The experiment was approved by the Ethics Committee of the University of São Paulo School of Philosophy, Sciences and Letters, Ribeirão Preto, Brazil.

\section{Material}

The experiment was performed during daylight hours in an isolated, soundproofed room at the central library on the Ribeirão Preto Campus. The room was lit with a $40 \mathrm{~lm} \mathrm{~W} \mathrm{~m}^{-1}$ compact fluorescent light bulb. Digital photographs of 20 abstract paintings were selected according to their pictorial compositions, themes, and artistic movements (Table 1). ${ }^{1}$

Table 1. List of paintings presented to the participants.

\begin{tabular}{l}
\hline Painting. Author (Artistic Movement) \\
\hline 1. Woman with a Guitar. Georges Braque (Cubism) \\
2. The Guitar Player. Pablo Picasso (Cubism) \\
3. Girl with Mandolin Fanny Tellier. Pablo Picasso (Cubism) \\
4. Wounded Bird and Cat. Pablo Picasso (Cubism) \\
5. Portrait of Pablo Picasso. Juan Gris (Cubism) \\
6. Nude Descending a Staircase. Marcel Duchamp (Cubism) \\
7. The Knife Grinder. Kazimir Malevich (Constructivism) \\
8. Abstract Speed. Giacomo Balla (Futurism) \\
9. Dynamism of a Dog on a Leash. Giacomo Balla (Futurism) \\
10. Lines of Movement and Dynamic Succession. G. Balla (Futurism) \\
11. Shape and Noise of Motorcyclist. Giacomo Balla (Futurism) \\
12. The Car Has Passed. Giacomo Balla (Futurism) \\
13. Girl Running on a Balcony. Giacomo Balla (Futurism) \\
14. The Hand of the Violinist. Giacomo Balla (Futurism) \\
15. Contemplating. Paul Klee (Expressionism) \\
16. Red Waistcoat. Paul Klee (Expressionism) \\
17. The Goldfish. Paul Klee (Expressionism) \\
18. Composition VIII. Wassily Kandinsky (Expressionism) \\
19. Number 8, 1949. Jackson Pollock (Abstract Expressionism) \\
20. Number 14, Grey. Jackson Pollock (Abstract Expressionism) \\
\end{tabular}

High-resolution artwork images were obtained from the Internet and were adjusted with regard to saturation, brightness, and size using Photoshop CS6 software. The final size of the images was approximately $30 \times 40 \mathrm{~cm}$ with respect to their vertical orientation. The artwork images occupied the central position of an LG screen that was connected to an HP notebook computer; the remainder of the screen was white.

Semantic 7-point Likert-type scales for Movement, Complexity, and Recognition and the 5-point SelfAssessment Manikin scale (Lang, 1980) were used to obtain information about how the painting compositions were perceived by the participants. Stimulus exposure, time estimation, and latency recordings were managed using the Wave Surfer program installed on the HP notebook computer.

\footnotetext{
${ }^{1}$ The cubist paintings used in this study consisted of the analytical phase of cubism in which the shape of the object is subjected to the two-dimensional surface of the canvas. The final result approaches abstraction (see Ganteführer-Trier \& Grosenick, 2004). Therefore, the abstract content of the cubists paintings that were used could be understood as a form of art that represents objects of our outer reality. They are composed of formal relations between colors, lines, and surfaces to render the reality of work in a non-representational way.
} 
Table 2. Means (standard deviations) of time estimation, latency, movement, arousal, recognition, and complexity for the six cubist paintings.

\begin{tabular}{cllllll}
\hline Painting & Time (s) & Latency (s) & Movement & Arousal & Recognition & Complexity \\
\hline 1 & $2.66(.64)-$ & $1.00(.60)$ & $2.23(1.23)$ & $1.62(.63)$ & $1.69(1.25)$ & $2.53(2.10)$ \\
2 & $2.88(.70)$ & $1.01(.70)$ & $3.23(1.83)$ & $2.69(.75)$ & $6.61(.76)$ & $2.69(1.70)$ \\
3 & $2.84(1.03)$ & $1.14(.74)$ & $3.00(1.52)$ & $2.23(.92)$ & $6.69(.48)$ & $3.38(1.85)$ \\
5 & $3.08(.64)$ & $.99(.30)$ & $2.76(1.23)$ & $2.07(1.18)$ & $6.84(.37)$ & $2.69(2.17)$ \\
6 & $3.65(1.30)+$ & $1.88(1.16)^{*}$ & $4.53(1.39)^{*}$ & $3.30(1.25) \#$ & $4.30(1.93)^{* *}$ & $4.30(1.65)$ \\
\hline
\end{tabular}

(-) Underestimated. (+) Marginally overestimated. $\left(^{*}\right) 1,2,3$ and $5<6$. (\#) $1<3,5$ and $6 ; 5<6 .\left(^{* *}\right)<7$ points.

\section{Procedure}

The participants individually entered the experimental room and were asked to sit in a chair. The tasks were orally explained to the participants. They were positioned facing the central region of the 19" LG monitor at a distance of approximately $60 \mathrm{~cm}$. They were instructed not to count time. The stimuli were presented by pressing the "presentation" key on the computer, and presentation was terminated after $3 \mathrm{~s}$. At this moment, the picture was replaced by a white screen, indicating that the participant could initiate time estimation. Immediately after each observation, the participant was instructed to reproduce the presentation duration by pressing the "initiate" key, which was finalized by pressing the "finished" key (reproduction method). The stimuli were randomly presented to the participants. The Wave Surfer program also recorded the latency between the end of each painting exposure and beginning of the participants' reproductions.

After the time estimations, the participants' were instructed to rate the amount of movement represented and complexity of the paintings on a 7-point scale. They were also asked to identify the figure depicted in the painting on a recognition scale and rate their level of arousal on a 5-point scale.

\section{Statistical analysis}

One-way repeated-measures analysis of variance (ANOVA) and the Student-Newman-Keuls post hoc test were used to compare time estimation, latency, movement, complexity, recognition, and arousal data for all of the paintings. One-way repeated-measures ANOVA and the Student-Newman-Keuls test were also used to compare the time estimation data according to the artistic movements and themes of the paintings between groups of paintings. The same analyses were used to compare the data of the different paintings in the paintings groups.

One-sample analyses were conducted to compare the mean time estimations of stimulus exposure with the actual time of exposure ( $3 \mathrm{~s})$. Also, the One-sample analyses were used to compare the data of recognition scale with 7 points. This analysis allowed inferences about the identification of the objects, animals, and human figures depicted in the paintings. Pearson correlation was employed to verify the association between Movement and Arousal scales.

\section{Results}

\section{Time estimations}

The ANOVA of the mean time estimations of the 20 paintings revealed significant differences between the $N u d e^{2}$ cubist painting by M. Duchamp and several other paintings $\left(F_{19,226}=1.77, p<.001\right)$. The duration of Nude exposure was estimated to be longer than the three futurist paintings by G. Balla (all $p<.05$ ), the abstract expressionist compositions by P. Klee $(p<.05)$ and J. Pollock $(p<.01)$, the expressionist painting by W. Kandinsky $(p<.01)$, and the cubist paintings that represented human figures by G. Braque $(p<.01)$, J. Gris $(p<.05)$, and P. Picasso $(p<.05)$. Significant differences were also found in latencies between the Nude painting and paintings that represented animals and abstract action paintings by Pollock $\left(F_{19,226}=2.27, p<.001\right)$, which were constructed as a function of real body movements performed by the artist (all $p<.05$; Table 2).

\section{Effects of arousal and movement measures on time perception}

The ANOVA of the Arousal and Movement data did not reveal a relationship between scores, indicating that implied movement and the emotional arousal evoked by the paintings were not directed related. This result was supported by Person correlation analyses. The analysis of Movement scores revealed that paintings with animal themes, such as Dynamism and Lines by Balla, received greater movement scores compared with the majority of the other paintings $\left(F_{19,265}=11.33, p<.001\right)$ including the Nude cubist painting by Duchamp that were overestimated (all $p<0.01$ ). The Dynamism and Lines paintings were not temporally distorted. Other paintings that were associated with different time estimations compared with Nude, including three futurist paintings by Balla, the abstract compositions by Pollock, and the expressionist painting by W. Kandinsky, did not receive different Movement scores compared with this cubist painting. However, Nude received greater movement scores than the two abstract expressionist compositions by Klee $(p<0.001)$ and cubist paintings that represented human figures by Gris and Picasso (both $p<.01$ ). Moreover, The Hand painting by Balla was not scored

${ }^{2}$ For simplicity, the first word of the name of each painting is used henceforth in the text. 
differently from Nude (both have the stroboscopic figurative representation of human movement) and it was not temporally distorted.

The ANOVA of Arousal scores showed that the 20 paintings were also scored differently $\left(F_{19,266}=5.09\right.$, $p<.001)$. These differences were smaller than those found in the Movement analyses. The Arousal scores were also not related to the time distortions observed. Nude received a greater arousal score than the abstract expressionist composition Contemplating by Klee and futurist painting Abstract Speed by Balla $(p<.01)$. The cubist painting of a cat (Wounded by Picasso) received lower arousal scores than Nude $(p<.05)$, but the other paintings that represented animals by Balla (Dynamism and Lines) were not scored differently from this cubist painting.

\section{Effect of painting themes and artwork schools on time perception}

Time and the other measures were also analyzed based on the paintings' themes and artistic schools of the artwork used. The ANOVA did not reveal differences between the mean time estimations among the cubist paintings group (Braque, Duchamp, J. Gris, and Picasso; paintings 1, 2, 3, 5, and 6), geometric futurist paintings group (Balla; paintings 8,11 , and 12), cubist expressionist and futurists paintings group that represented animals (Balla, Klee, and Picasso; paintings 4, 9, 10, and 17), geometric expressionist paintings group (Kandinsky, Klee, and Pollock; paintings 15, $16,18,19$, and 20), and futurist paintings group that represented human body parts (K. Malevich and Balla; paintings 7,13 , and 14 ).

The data analyses showed that only the five cubist paintings that represented human forms (paintings 1,2, $3,5$, and 6$)$ had different time estimations $\left(F_{4,56}=3.85\right.$, $p<.01)$. The post hoc comparisons showed that all of the paintings had shorter time estimations than Nude (i.e., the painting with stroboscopic representation of a human figure; $p<.05$ ). Student's $t$-test showed that the time of Woman presentation was underestimated $\left(t_{15}=\right.$ $-2.37, p<.05)$, and Nude was marginally overestimated $\left(t_{15}=1.81, p<.08\right)$ compared with the actual exposure time of $3 \mathrm{~s}$ (Table 2). The mean latency scores were also statistically different between these paintings $\left(F_{4,56}=\right.$ $6.09, p<.001)$. Nude had a greater latency compared with the other cubist paintings (all $p<.01$ ).

Because time estimations were verified only for these cubist paintings that represented human figures, further analyses considered the differential semantic scales only for these paintings. The Movement data analysis showed that these cubist paintings were scored differently $\left(F_{4,56}=6.59, p<.001\right)$. Nude received higher movement scores than the other paintings (all $p$ $<.01)$. Arousal scores were also different among these paintings $\left(F_{4,56}=5.93, p<.001\right)$. Nude received a higher arousal score than the Woman and Portrait paintings (all $p<.001$ ). Woman had a lower arousal score than
Girl and Portrait, indicating that implied movement and arousal scores were not related $(p<.01)$.

Further analyses evaluated the complexity and recognition measures among these cubist paintings (paintings 1, 2, 3, 5, and 6). The ANOVA of complexity scores did not reveal significant differences among these five cubist paintings. However, the paintings received different recognition scores $\left(F_{4,56}=6.09, p<.001\right)$. Considering that a score of 7 points indicated verified identification of a human figure, the $t$-test showed that The Guitar $\left(t_{15}=15.30, p<.01\right)$ and Nude $\left(t_{15}=5.02\right.$, $p<.01)$ were less easily identified as human images compared with the other three paintings.

\section{Discussion}

The results of this experiment can be discussed in terms of the observation time of different artworks in a museum. For example, when a person goes to a museum to appreciate a permanent collection, he/she is exposed to different pieces of artwork, and the time spent observing each one depends on their appraisal characteristics that demand more than $3 \mathrm{~s}$ of observation. Therefore, interest and pleasure while viewing a specific painting could be related to how long a person observes it. Thus, the results for the 20 paintings used in Experiment 1 should be discussed by considering the guidelines of the new experimental aesthetics (Berlyne, 1974) taking into account the visual exploration involved in artistic appreciation.

In this experiment, paintings with different themes and from different artistic schools were observed for only $3 \mathrm{~s}$. The results showed that different paintings caused time distortions, independent of their abstract compositions. These results are consistent with Stoyanova, Yakimoff, Gourevich, and Mitrani (1987) who reported that figurative and abstract paintings that were presented for short intervals (.6-1.5 s) also distorted time perception. These authors indicated that under- and overestimations of time were caused by the complexity of the artwork, but they did not discuss which pictorial features were responsible for these time distortions.

Based on the present results, we can make inferences about the effects of each painting on subjective time because the data were analyzed by considering the paintings' specific themes and schools. Only the cubist paintings that represented human figures were estimated differently when they were analyzed together. Interestingly, the five cubist paintings that represented human forms were mostly brown, ocher, and green. Representing the analytical phase of cubism, they were nearly monochromatic, which allowed analysis of form without distraction by colors (Ganteführer-Trier \& Grosenick, 2004; Strickland, 2004). However, they differ with regard to the amount of pictorial abstraction that results from different three-dimensional planes of the human figure representation, which causes distinct interpretative ambiguities.

Nicki, Lee, and Moss (1981) showed that subjects observed cubist paintings for a longer time according to 
an increase in the subjective ambiguity of the paintings. These authors suggested that interest and pleasure in a painting are associated with identification of an object or person that is represented in the artwork. Furthermore, Kuchinke, Trapp, Jacobs, and Leder (2009) instructed subjects to indicate the moment at which they recognized any object in several cubist paintings. The paintings with high content accessibility were processed faster toward the point of explicit classification of the depicted content. Therefore, it is possible to pointed out that the five cubist paintings used in the present study affected time perception according to affective aesthetic responses in the observer that depended on how ease they were processed.

In the present study, all of the cubist paintings that represented human forms received the same scores on the complexity scale. However, Woman and Nude were less easily associated with human images on the recognition scale. These two paintings were also scored differently on the movement scale and were under- and overestimated, respectively, compared with the actual exposure time $(3 \mathrm{~s})$. These data could explain why the stroboscopic painting Nude, which implies more movement, had a longer time estimation than the other cubist paintings, such as Woman (which implies less movement), that do not represent intentional human movements (see Table 2). Furthermore, Hekkert and van Wieringen (1990) used the rate of identification to evaluate the time required for subjects to recognize human figures in cubist paintings. The results showed that Nude was recognized after $7.5 \mathrm{~s}$, whereas Portrait was quickly identified $(<1 \mathrm{~s})$. Paintings with compositions similar to Woman were recognized after $>9.5 \mathrm{~s}$. These authors suggested that aesthetic pleasure is related to the ability to recognize human figures, independent of the paintings' complexity. Notably, Nude had the highest latency. This result could be interpreted as a longer processing time compared with other cubist paintings (Table 2).

Nude received the highest movement score and was marginally overestimated. Similarly, Nather et al. (2011) showed that the duration of exposure to figurative human images (i.e., Degas' ballerinas) was marginally distorted when 2-8 s durations of stimulus exposure were used. These authors found that the magnitude of time distortions was greater with shorter exposures $(<1 \mathrm{~s})$ in which body postures that imply more movement were judged with more arousal through embodiment mechanisms. Therefore, it is possible to infer that the same mechanisms of time estimation may be required when people observe abstract or figurative human figures that represent movements of different types and intensities such as the stroboscopic artwork.

Different pictorial characteristics of cubist paintings were analyzed in previous studies (Hekkert \& van Wieringen, 1990; Kuchinke et al., 2009; Nicki et al., 1981). These studies reported that the duration of exposure is a significant factor in figurative recognition and determines interest in and pleasure evoked by these paintings. Implied movement is an intrinsic part of their visual processing, and evaluating the role of movement perception in time distortion was important. In the present study, all of the cubist paintings received the same complexity scores. Figurative forms were not easily recognized for only two paintings: Nude and The Guitar. Nude (which implies more movement) was not scored differently from The Guitar (which implies less movement) in the arousal measure, and the durations of exposure were over- and underestimated, respectively. The data from Experiment 1 did not allow definitive conclusions about whether the implied movement in the paintings was the unique parameter that was responsible for the time distortions observed, and emotional arousal may have played a role.

To further investigate the effects of implied movement associated with arousal levels and embodiment mechanisms, four cubist paintings were presented for 3 and $9 \mathrm{~s}$ to different groups of participants in Experiment 2. These different durations tested the transient effects of arousal that occur with stimulus exposure times of 3-4 s.

\section{EXPERIMENT 2}

\section{Methods}

The participants were undergraduate students from the University of São Paulo, Ribeirão Preto, Brazil, and randomly invited to participate in the study. They were untrained in visual arts and had normal or corrected-tonormal vision. This experiment was approved by the Ethics Committee of the University of São Paulo School of Philosophy, Sciences and Letters in Ribeirão Preto, Brazil.

\section{Groups and procedure}

The methods of Experiment 2 were basically the same as in Experiment 1, with the exception that only four cubist paintings that represented human figures were used. These paintings were selected because two of them were previously reported to be recognized as figurative forms after $7.5 \mathrm{~s}$ (Hekkert \& van Wieringen, 1990), and they were temporally distorted compared with the other cubist paintings (Experiment 1). The arousal and movement scores for three of these paintings were not different. Nude (painting 6) had higher arousal and movement scores than the other cubist paintings, and its exposure time was overestimated. In contrast, the other three paintings did not receive different arousal or movement scores, and the exposure time for Woman (painting 1) was underestimated.

Four groups of participants were used. They were defined according to the three cubist paintings used (GA3, GA9, GB3, and GB9 groups). The GA3 and GA9 groups were exposed to Woman (painting 1), Girl (painting 3), and Nude (painting 6). These paintings were presented to 50 participants: $3 \mathrm{~s}$ in the GA3 group (23 individuals, 10 men; mean age, 21.30 years, $\mathrm{SD}=$ 3.49 years) and $9 \mathrm{~s}$ in the GA9 group (27 individuals, 13 men; mean age $=20.00$ years, $\mathrm{SD}=2.26$ years). 
The GB3 and GB9 groups were exposed to The Guitar (painting 2), Girl (painting 3), and Nude (painting 6; Table 3). These paintings were presented to 60 participants: $3 \mathrm{~s}$ in the GB3 group (28 individuals, 19 men; mean age $=21.25$ years, $\mathrm{SD}=1.92$ years) and 9 $\mathrm{s}$ in the GB9 group (32 individuals, 19 men; mean age, 20.81 years, $\mathrm{SD}=2.19$ years $).{ }^{3}$ Notice that two paintings were the same (Nude and Girl) in all of the groups. Similar to Experiment 1, the images were presented in random order for the four groups.

Semantic Differential Arousal and Movement Scales were used in this experiment. They were completed after the time estimations according to the procedure adopted in Experiment 1.

\section{Statistical analysis}

One-way repeated-measures ANOVA and the Student-Newman-Keuls post hoc test were used to compare time estimations and latency data for each group separately (GA3, GA9, GB3, and GB9). Onesample analyses were conducted to compare the time estimations in the GA3, GA9, GB3, and GB9 groups with the actual exposure times ( 3 or $9 \mathrm{~s}$ ).

Two-way not repeated-measures ANOVA and the Student-Newman-Keuls post hoc test were used to analyze the Movement and Arousal scale data between the GA3 and GA9 groups and between the GB3 and GB9 groups. Additional Two-way repeated-measures ANOVA and the Student-Newman-Keuls post hoc test were used to analyze the Movement and Arousal scale data between the GA3 and GA9 groups together (GA3/9) and between the GB3 and GB9 groups together (GB3/9). This procedure was used because the data obtained using semantic scales were obtained after the time estimations, and the participants observed the artworks for however long they wanted. Pearson correlation was employed to verify the association between Movement and Arousal scales.

Student $t$-test was used to compare the latency data for each painting between the GA3 and GA9 groups and between the GB3 and GB9 groups separately.

\section{Results}

\section{Time measures}

The ANOVA of the time estimation data did not reveal significant differences between the GA3 and GB3 groups (Table 3). The one-sample analysis showed that the time estimations for the paintings were not different from the actual $3 \mathrm{~s}$ exposure time in the GA3 and GB3 groups.

Time distortions were observed when the paintings were presented for $9 \mathrm{~s}$ in the GA9 and GB9 groups.

\footnotetext{
${ }^{3}$ These paintings are available on the following websites: Woman (www.wikipaintings.org/en/georges-braque/womanwith-a-guitar-1913), The Guitar (www.wikipaintings.org/en/ pablo-picasso/guitar-player-1910), Girl (www.wikipaintings. org/en/pablo-picasso/girl-with-mandolin-fanny-tellier-1910), and Nude (www.wikipaintings.org/en/marcel-duchamp/nudedescending-a-staircase-no-2-1912).
}

Interestingly, the ANOVA showed that these time distortions were not the same in both groups. In the GA9 group, differences in time estimations were not observed among the paintings. However, in the GB9 group, Nude (painting 6) and Girl (painting 3), both of which imply more movement, the exposure times were estimated to be longer compared with The Guitar (painting 2; which implies less movement; $F_{2,62}=4.22$, $p<.01)$. The one-sample analysis showed that the exposure time for Nude was overestimated in both the GA9 and GB9 groups relative to the actual exposure time of $9 \mathrm{~s}\left(t_{26}=2.15, p<.05\right.$, and $t_{34}=2.18, p<.05$, respectively).

The ANOVA did not reveal significant differences in latency among the paintings that were presented for 3 or $9 \mathrm{~s}$ (GA3 and GB9 and GB3 and GB9 groups, respectively; Table 3). Comparisons of the data for each painting separately showed that the latency was greater for $9 \mathrm{~s}$ exposure times for the most abstract paintings (Nude and Woman) in the GA3 and GA9 groups $\left(t_{48}=-2.93, p<.001\right.$, and $t_{46}=-2.65, p<.01$, respectively) and for Nude and The Guitar in the GB3 and GB9 groups $\left(t_{58}=-2.10, p<.05\right.$, and $t_{57}=-1.94, p$ $=.05$, respectively).

\section{Arousal and movement measures}

The ANOVA did not reveal differences in arousal and movement scores between the GA3 and GA9 groups or between the GB3 and GB9 groups. These results were expected because these data were obtained after time estimation. The participants finished the task of completing the semantic scales after the time estimations and observing the paintings for as long as they wanted. The ANOVA showed that the Arousal scores were different between GA3 and GA9 together $(\mathrm{GA} 3 / 9)$ and GB3 and GB9 together (GB3/9) $\left(F_{2,98}=\right.$ $25.21, p<.001$, and $F_{2,118}=17.39, p<.001$, respectively; Table 3): Nude (3.5 points, approximately) received higher arousal scores than the other paintings (2.5 points, approximately) (all $p<.01$ ).

The ANOVA showed that the Movement scores were different between the GA3 and GA9 groups together $\left(\mathrm{GA} 3 / 9 ; F_{2.98}=10.21, p<.001\right)$ and the $\mathrm{GB} 3$ and GB9 groups together $\left(\mathrm{GB} 3 / 9 ; F_{2,118}=25.08, p<.001\right)$. Woman (3.46 points) received lesser movement scores than Girl (4.22 points) and Nude (4.96 points) paintings in the GA3/9 (both $p<.05$ ). The same was observed in the GB3/9: Nude and Girl paintings (4.48 and 4.74 points, respectively) received greater movement scores than The Guitar painting (2.76 points) (both $p<.01$ ). Notice that movement scores for Nude and Girl were 1.5 points higher than for The Guitar in the GB3 and GB9 groups together (GB3/9), but movement scores in the GA3 and GA9 groups together (GA3/9) were $\leq 1.5$ points (Table 3).

Pearson correlation analyses did not reveal correlation between Arousal and Movement scores, indicating that implied movement and the emotional arousal evoked by the paintings were not directed related. 
Table 3. Mean \pm standard deviation of time estimation, latency, movement, arousal, recognition, and complexity for the four cubist paintings in GA and GB groups.

\begin{tabular}{lllllll}
\hline \multicolumn{7}{l}{ GA3/9 paintings } \\
Parameter & $\mathbf{1}$ & $\mathbf{3}$ & $\mathbf{6}$ & $\mathbf{2}$ & $\mathbf{3}$ & $\mathbf{6}$ \\
\hline Time (3 s) & $3.28 \pm .80$ & $3.04 \pm 1.00$ & $2.90 \pm .65$ & $3.04 \pm .60$ & $3.22 \pm .71$ & $3.19 \pm .82$ \\
Time (9 s) & $9.13 \pm 2.87$ & $9.81 \pm 2.43$ & $9.82 \pm 2.01^{*}$ & $8.96 \pm 2.11$ & $9.55 \pm 2.62^{\mathrm{L}}$ & $11.13 \pm 5.19^{* \mathrm{~L}}$ \\
Latency (3 s) & $1.21 \pm .39$ & $1.37 \pm .67$ & $1.28 \pm .48$ & $1.52 \pm .64$ & $1.82 \pm 1.10$ & $1.49 \pm .74$ \\
Latency (9 s) & $1.65 \pm 1.02^{+}$ & $1.71 \pm 1.16$ & $1.93 \pm 1.66^{+}$ & $2.69 \pm 1.57^{+}$ & $2.44 \pm 1.39$ & $2.08 \pm .79^{+}$ \\
Arousal (3/9 s) & $2.50 \pm .95$ & $2.52 \pm 1.01$ & $\mathbf{3 . 7 6} \pm \mathbf{1 . 0 6}$ & $2.55 \pm .92$ & $2.60 \pm 1.01$ & $\mathbf{3 . 4 6} \pm \mathbf{1 . 0 9}$ \\
Movement (3/9 s) & $3.46 \pm 1.86$ & $4.22 \pm 1.82 \#$ & $\mathbf{4 . 9 6} \pm \mathbf{1 . 9 4}$ & $2.76 \pm 1.72$ & $\mathbf{4 . 4 8} \pm \mathbf{1 . 7 5}$ & $\mathbf{4 . 7 4} \pm \mathbf{2 . 1 7}$ \\
\hline
\end{tabular}

* Overestimation. ${ }^{\mathrm{L}}$ Paintings 3 and 6 were estimated longer than painting 2 in the GB9 group. + Longer latency for $9 \mathrm{~s}$ compared with $3 \mathrm{~s}$ exposure. Italics indicate that the paintings received different scores for arousal and movement relative to the paintings marked in bold. \# Painting 3 was also scored with less movement than painting 1 in the GA3/9 groups.

\section{Discussion}

Experiment 2 showed that time perception was affected by cubist paintings that represented human figures when they were presented for $9 \mathrm{~s}$ to different groups of participants (GA9 and GB9 groups). This experiment also revealed a contextual effect of artwork groupings on time estimation. Woman, Girl, and Nude were not estimated differently in the GA9 group. However, in the GB9 group, Nude and Girl were estimated longer than The Guitar. Moreover, Nude was overestimated in both the GA9 and GB9 groups. In fact, this overestimation could be associated with the arousal scores for Nude. This painting received greater arousal scores than the other paintings (Table 3). However, Girl received the same arousal score in both the GA9 and GB9 groups and was estimated longer than The Guitar in the GB9 group. However, the same was not verified for this painting in the GA9 group, in which all of the paintings were similarly estimated.

The different time estimations found between the GA9 and GB9 groups could be better understood by relating them to the implied movement scores. Nude and Girl (which imply more movement) were estimated longer than The Guitar (which implies less movement) in the GB9 group because of their implied movement. Moreover, an explanation was less evident for the time estimations among the three paintings in the GA9 group. A more refined analysis of the effect of movement scores on time perception clarified these different results. The paintings were not the same in both groups as well as their movement scores. Movement scores among Woman (3.46), Girl (4.22), and Nude (4.96) paintings were $\leq 1.5$ points in the GA3 and GA9 groups together (GA3/9), and the paintings were not estimated differently. In contrast, in the GB3 and GB9 groups together (GB3/9), the difference among The Guitar (2.76), Girl (4.48), and Nude (4.74) paintings were > 1.5 points (Table 3 ). Therefore, Nude and Girl were estimated longer than The Guitar in the GB9 group due to the implied movement among these paintings.

These differences in time estimation are consistent with the results of a previous study that used figurative artworks that represented human bodies that performed actions (Nather \& Bueno, 2011). These authors used different photographs of ballerina sculptures by Edgar Degas that were presented to three different groups of participants, similar to the present experimental design (three images in each group). Using a long duration of exposure (36 s) and grouping the same images according to their movement scores $(1.5,3.0,4.5$, and 6.0 points), these authors showed that participants estimated the time of exposure similarly for ballerina bodies that had implied movement scores $\leq 1.5$ points. Altogether, these data corroborate the present results, in which the images in the GB9 group were estimated differently based on their movement scores ( $>1.5$ points). This was not observed in the GA9 group in which the movement scores were $\leq 1.5$ points. Therefore, the results of these studies suggest that the contextual grouping of images affects the processes that underlie both visual movement and time perception.

The participants' latencies were another important aspect of this study, which could reflect an affect of the visual processing of the artwork on time perception. The latencies in the GA3, GB3, GA9, and GB9 groups were not different. However, the latencies were different between the more abstract cubist paintings (The Guitar, Woman, and Nude) when they were presented for $9 \mathrm{~s}$. Aesthetic pleasure in cubist paintings is related to the ability to recognize figurative forms in short (milliseconds) and long (9.5 s or more) exposures (Hekkert \& van Wieringen, 1990). Additionally, arousal has no effect with exposure times $>9 \mathrm{~s}$, independent of the transient effect of arousal at 3-4 s, indicating that other cognitive processes mediate time estimations with $9 \mathrm{~s}$ exposure times (see also Elbert et al., 1991) such as movement perception.

Finally, these paintings were presented with other abstract paintings in Experiment 1. In Experiment 2, only four mostly similar cubist paintings that represented human forms from the analytical phase of cubism were presented to the participants. These four paintings are more easily comparable with regard to their cognitive appraisals. This can explain why Nude and Woman were marginally overestimated and underestimated in Experiment 1 with $3 \mathrm{~s}$ exposure. The same was not 
observed in the GA3 and GB3 groups with $3 \mathrm{~s}$ exposure in Experiment 2.

\section{General discussion}

Subjective time was affected by abstract paintings that were presented for different durations (3 and 9 s). These time distortions may be related to different psychological processes that involve interactions between the observation durations of the two experimental exposure conditions: (i) 20 paintings with different themes from different artistic schools (cubism, expressionism, and futurism) that were presented for $3 \mathrm{~s}$ (Experiment 1) and (ii) four cubist paintings with the same theme that were presented for $9 \mathrm{~s}$ (Experiment 2). Considering the artwork schools and themes of the paintings, the time estimation results indicate that cubist paintings that are composed of abstract human forms differentially distorted timing depending on the groups' exposure times ( 3 and $9 \mathrm{~s}$ ). The time estimation data were more related to implied movement than to emotional arousal evoked by the paintings that were presented for $9 \mathrm{~s}$, likely because cognitive appraisal affects time processing. Therefore, Experiments 1 and 2 highlight the importance of artwork grouping and the duration of observation, which was more evident for the cubist paintings that represented human forms. However, why did these cubist paintings differentially affect time perception when they were presented for different times and in different experimental groupings?

In Experiment 1, the participants estimated time with very different artworks, and comparisons were made with their cognitive appraisals. Several implicit aspects of an aesthetic episode were processed together to generate different effects on time perception that were attributable to the possible comparisons established with observations of all 20 paintings. Woman was underestimated and Nude was marginally overestimated relative to the actual exposure time of $3 \mathrm{~s}$. Nude was estimated longer than Woman. Interestingly, all of the cubist paintings in this experiment were not different in the complexity measure. These two paintings were less easily recognizable as a human figure compared with the other cubist paintings (recognition measure). Furthermore, they were scored differently in the movement and arousal measures.

In Experiment 2, Nude was overestimated in both the GA9 and GB9 groups relative to the actual exposure time of 9 s. Girl, which did not affect time perception in Experiment 1, caused time distortion in one group in Experiment 2. This aspect of time perception (i.e., a grouping effect) can be understood by considering the movement and arousal measures. The estimations of the Nude and Girl (which imply more movement) exposure times were longer compared with The Guitar (which implies less movement) in the GB9 group. But the same was not observed in the GA9 group. Estimations of the Nude and Girl exposure times were not longer compared with Woman, and they were scored similarly in the movement measure. Only Nude received a greater arousal score in Experiment 2. When people observe these and other paintings in a museum (i.e., an ecological context), interest and pleasure in one specific painting demand different processing times because the subjects are freely attentive to different aspects of the artwork and not subjected to the elapsed time of exposure. Therefore, this aspect of visual artwork appreciation could be available in terms of how long a person observes and comprehends it.

The cubist paintings that represented human forms were the same in both experiments, and they were relatively similar in their pictorial compositions, characterized by similar elements with regard to the amount of pictorial abstraction (Ganteführer-Trier \& Grosenick, 2004). Woman, The Guitar, and Nude were not easily related to a human figure, demonstrating that their figurative content was not quickly distinguished. This result could be related to previous studies that used only cubist paintings. Nicki et al. (1981) showed that subjects observed cubist artworks for more time because of an increase in their subjective ambiguity as the identification of a human figure in the compositions. Cubist paintings with higher content accessibility (i.e., less ambiguity) could be processed faster. This aspect of visual perception is an important feature of an aesthetic episode (Cupchick \& Gebotys, 1998). Therefore, the recognition responses in the observer depend on how easily they were processed over time (see also Kuchinke et al., 2009). Moreover, Hekkert and van Wieringen (1990) showed that more than $3 \mathrm{~s}$ was necessary to identify a figurative form in cubist paintings. Using an identification rate to evaluate the time required for subjects to recognize human figures in cubist paintings, they showed that Nude was recognized after 7.5 s. A similar painting composition, such as Woman, was recognized after $9.5 \mathrm{~s}$. Therefore, the two exposure durations used in the present study ( 3 and 9 s) were essential to explain the apparently discrepant time estimation data obtained in both experiments. In Experiments 1 and 2 (with $3 \mathrm{~s}$ exposure), these cubist paintings could be interpreted as only abstract compositions that do not necessarily involve human forms because of the short duration of exposure (3s), which is insufficient for the participants to comprehend and identify human forms or imply movement. Thus, in Experiment 2 (9 s exposure groups), Nude was better understood as a human form or a person who is performing a movement. In contrast, $9 \mathrm{~s}$ exposure to Woman and The Guitar did not allow the observers to recognize abstract human forms or perceive movement.

This aspect (observation time) of abstract artwork was also evaluated according to the latency measure. The latencies among the paintings were the same when the different cubist paintings were presented for 3 or 9 $\mathrm{s}$ (Experiment 2). However, an interesting latency result was found when comparisons were made between the 3 and $9 \mathrm{~s}$ exposure groups. The more abstract paintings produced longer latencies when presented for $9 \mathrm{~s}$, 
including Nude and Woman in the GA9 group and Nude and The Guitar in the GB9 group (see Table 3). In contrast, the same was not observed for Girl. Despite its implicit human form abstraction, Girl was recognized as a woman figure. Therefore, these results could be interpreted as indicative of image comprehension and processing. The participants may have been attempting to understand the image content before making time estimations, which could affect their further subjective time estimation when the duration of exposure was $9 \mathrm{~s}$ (Experiment 2). This aspect of time perception requires further investigation because it was used as a control measure relative to time perception in this study, but it may provide a possible explanation for the subjective time perception results.

Exposure time was a relevant aspect of time perception when the same impressionist art pieces that represented figurative human bodies and implied different body movements were studied (Nather, Bueno, $\&$ Bigand, 2013). Time duration in an aesthetic episode can surpass the effects of other empathic properties of artworks. For example, the time of exposure to the same ballerina image that implied movement (a great arabesque) was overestimated when presented for $36 \mathrm{~s}$, but the same was not observed when it was presented for $18 \mathrm{~s}$. This information may clarify the different time distortions observed between the 3 and 9 s exposure times because of the emotional arousal elicited by cubist paintings. Nude received greater arousal scores than the other three paintings. However, the arousal effects in the observer could be transient with exposure longer than $3 \mathrm{~s}$ in which high arousal levels are more effective in the early phase (2 s) of time processing (Angrili et al., 1997; Nather et al., 2011). Therefore, with $9 \mathrm{~s}$ exposure to the paintings, time distortions were unrelated to emotions but rather to determined properties of its composition that could be related, for example, to empathy and embodiment mechanisms evoked by artworks that represent motion (Freedberg \& Gallese, 2007).

Gil and Droit-Volet (2012) discussed the fundamental role of arousal related to the emotional content of human pictures based on the Affective Picture System (Lang, Bradley, \& Cuthbert, 2008). They showed a lengthening effect on the duration of emotional pictures compared with neutral ones, indicating that the magnitude of this effect increases with greater levels of arousal and impact of emotional pictures. For example, the duration of disgust-inducing pictures (e.g., body mutilation) was judged to be longer than fear-inducing pictures (e.g., snake). The impact of artwork appreciation, such as with cubist paintings, is not the same as with real images of humans in dangerous or fearful contexts (Grommet, Droit-Volet, Hemmes, Baker, \& Brown, 2008). For example, excluding arousal levels, phobic subjects make longer estimates of $45 \mathrm{~s}$ observation of a spider (Watts \& Sharrock, 1984). Arousal may be an important mechanism that mediates the effect of emotion on time perception. In this study, however, it must also be considered in terms of hedonic effects and cognitive appraisals that are related to the different properties of the artwork used (e.g., Silvia, 2005a, b; Russell, 2003).

From this perspective, the present study corroborated the transient effect of emotional arousal on time perception when the duration of an aesthetic episode is greater than 3-4 s (see also Lambrechts, Mella, Pouthas, $\&$ Noulhiane, 2011) through embodiment mechanisms that are related to the kind of represented movement, either abstract or not. Therefore, time distortions for 9 s exposure (Experiment 2) were related to movement perception in which the paintings received both greater arousal and movement scores. Additionally, the arousal scores were relatively similar among the groups of participants in Experiment 2, but movement scores were different (see Table 3). Thus, the different movement scores among the paintings must be considered. When movement scores were $\leq 1.5$ points, the estimations of the paintings' exposure times were not different. However, the estimations of the paintings' exposure times were different when the movement scores were $>$ 1.5. The exposure time for The Guitar (3.0 points, approximately) was estimated to be shorter compared with Nude and Girl (4.5 to 5.0 points, approximately). These results support the hypothesis proposed by Nather and Bueno (2011) in which movement scores $>1.5$ points are sufficient to produce time distortions. A previous study used long-duration exposure (36 s) to different photographs of ballerina sculptures that were presented to three different groups of participants, similar to the experimental design in the present study (three images per group). Therefore, different kinds of represented movement in figurative and abstract artwork affect time perception. The stroboscopic movement of Nude was overestimated because the attention evoked (see Elbert et al., 1991) by this artwork when it was presented for $9 \mathrm{~s}$ allowed the individuals to compare paintings with similar characteristics from the same artistic school.

The activation of embodiment mechanisms encompasses the simulation of actions, emotions, and corporeal sensations by motion-sensitive cortical brain areas (Freedberg \& Gallese, 2007), which may explain the time distortions. Differential activation of the MT+ area and other cortical brain areas have been discussed in studies that used Manga drawings and Van Gogh paintings (Osaka, Matsuyoshi, Ikeda, \& Osaka, 2010; Thakral et al., 2012; Vartanian \& Goel, 2004). According to Kim and Blake (2007), different abstract paintings from different schools, such as Nude, imply different movement intensities and activate the MT+ brain area. In this study, the participants were previously exposed to the abstract paintings that were used. In another study that used functional magnetic resonance imaging, the latencies were significantly shorter when the subjects were previously trained to recognize represented objects in cubist paintings (Wiesmann \& Ishai, 2010). Therefore, the different time estimation results between the 3 and $9 \mathrm{~s}$ exposures to the paintings revealed that embodiment mechanisms, through empathic processes, were more 
effective when the participants were able to better understand these abstract artistic compositions. This can be established with exposure times $>7.5 \mathrm{~s}$ (see $\mathrm{Nude}$ painting results in Hekkert \& van Wieringen, 1990). More than emotional arousal, understanding the implicit actions of these abstract human forms through empathy enabled individuals to recognize and make comparisons based on memories of the elapsed time to perform the observed (stroboscopic) movements, such as a person descending a staircase. Therefore, overestimations for Nude were consistent with the accumulation of internal clock pulses. More attention was given to this painting because of the inherent implicit movement representation (i.e., a stroboscopic image). The internal clock model supposes that timing is derived from the number of pulses generated by one pacemaker linked to one accumulator (Gibbon, Church, \& Meck, 1984). Attention modulates the accumulation of pulses that are stored in the accumulator, acting as a switch (see also Zakay \& Block, 1996). Because of the fact that the human brain is able to perceive different implied movements of different artistic representations (Cutting, 2002), the switch in attention increases the number of accumulated pulses during the observation of this kind of movement (i.e., stroboscopic) such that exposure time is judged to be longer. The greater implied movement in this abstract painting then accelerates the pulses of the internal marker, thus generating overestimations. The same was not observed for the other cubist paintings because they do not objectively represent movement. The internal clock runs faster with embodied movement associated with perceived motion when the duration of exposure is sufficient and considering the cognitive appraisals that are involved in an aesthetic episode (e.g., people observing abstract cubist paintings). The latency data confirmed this hypothesis, in which the latencies were different when the subjects observed these paintings for 3 and $9 \mathrm{~s}$. The latency was higher when Nude was observed for $9 \mathrm{~s}$, indicating that the duration of an aesthetic episode affects the subjective perception of time, independent of its cognitive possessing.

\section{Conclusion}

Time perception in artwork episodes depends on several factors that are implicitly related to the combination of image observation, duration of exposure, and processing. The experiments showed that time distortions were more attributable to implied movement perception than to the arousal evoked. Therefore, visual movement perception in static artwork accentuates the effects of arousal (emotions) when exposure times are sufficient to comprehend an artwork. Implied movement in two-dimensional artworks (e.g., paintings) is an important factor of visual perception that affects timing. Further research that uses abstract artwork from different schools (e.g., artwork with proven emotional content, such as The Scream by Edgard Munch) may provide information about other emotional aspects and processes that are related to time perception and embodiment when subjects observe paintings that represent human figures that are or are not in motion.

\section{Acknowledgements}

Francisco C. Nather received a Young Investigators Award from the São Paulo Research Foundation (FAPESP). José Lino Oliveira Bueno received a Research Grant from the National Counsel for Technological and Scientific Development (CNPq). Paola Alarcon Monteiro Fernandes received a PIBIC/ Santander Undergraduate Research Scholarship.

\section{References}

Angrili, A., Cherubini, P., Pavese, A., \& Manfredini, S. (1997). The influence of affective factors on time perception. Perception and Psychophysics, 59, 972-982.

Berlyne, D. E. (1974). Studies in the new experimental aesthetics: steps toward an objective psychology of aesthetic appreciation. New York: Wiley.

Braddick, O. (1995). The many faces of motion perception. In: R. L. Gregory, J. Harris, P. Heard, \& D. Rose (Eds.), The artful eye (pp. 205-231). Oxford: Oxford University Press.

Cupchick, G. C. (1976). Perspective théorique et empirique sur peintures impressioniste. Bulletin de Psychologie, 36, 720-729.

Cupchick, G. C., \& Gebotys, R. (1988). The experience of time, pleasure, and interest during aesthetic episodes. Empirical Studies of the Arts, 6, 1-12.

Cutting, J. E. (2002). Representing motion in a static image: constraints and parallels in art, science, and popular culture. Perception, 31, 1165-1193.

Elbert, T., Ulrich, R., Rockstroh, B., \& Lutzenberger, W. (1991). The processing of temporal intervals reflected by CNV-like brain potentials. Psychophysiology, 28, 648-655.

Freedberg, D., \& Gallese, V. (2007). Motion, emotion and empathy in esthetic experience. Trends in Cognitive Sciences, 11, 197-201.

Ganteführer-Trier, A., \& Grosenick, U. (2004). Cubism. Koln: Tachen.

Gibbon, J., Church, R. M., \& Meck, W. H. (1984). Scalar timing in memory. In: J.A. Gibbon, \& L. Allan (Eds.), Timing and time perception (series title: Annals of the New York Academy of Sciences, vol. 423, pp. 52-77). New York: New York Academy of Sciences.

Gil, S., \& Droit-Volet, S. (2012). Emotional time distortions: the fundamental role of arousal. Cognition and Emotion, 26, 847-862.

Grommet, E. K., Droit-Volet, S., Gil, S., Hemmes, N. S., Baker, A. H., \& Brown, B. L. (2011). Time estimation of fear cues in human observers. Behavioural Processes, 86, 88-93.

Growe, B. (2001). Degas. Alemanha: Taschen.

Hekkert, P., \& van Wieringen, P. C. (1990). Complexity and prototypicality as determinants of the appraisal of cubist paintings. British Journal of Psychology, 81, 483-495.

Kim, C. Y., \& Blake, R. (2007). Brain activity accompanying perception of implied motion in abstract paintings. Spatial Vision, 20, 545-560.

Kourtzi, Z., \& Kanwisher, N. (2000). Activation in human MT/ MST by static images with implied motion. Journal of Cognitive Neuroscience, 12, 48-55.

Kourtzi, Z. (2004). "But still, it moves." Trends in Cognitive Sciences, 8, 47-49.

Kuchinke, L., Trapp, S., Jacobs, A. M., \& Leder, H. (2009). Pupillary responses in art appreciation: effects of aesthetic emotions. Psychology of Aesthetics, Creativity, and the Arts, 3, 156-163.

Lambrechts, A., Mella, N., Pouthas, V., \& Noulhiane, M. (2011). Subjectivity of time perception: a visual emotional orchestration. Frontiers in Integrative Neuroscience, 5, 73.

Lang, P. J. (1980). Behavioral treatment and bio behavioral assessment: computer applications. In: J. B. Sidowski, \& J. H. Johnson (Eds), Technology in mental health care delivery systems (pp. 119-137). Norwood, NJ: Ablex.

Lang, P. J., Bradley, M. M., \& Cuthbert, B. N. (2008). International Affective Picture System (IAPS): affective ratings of pictures and instruction manual. Gainesville, FL: University of Florida. 
Martinez-Conde, S., \& Macknik, S. L. (2010). Art as visual research: 12 examples of kinetic illusions in Op Art. Scientific American, 20, 48-55.

Nather, F. C., \& Bueno, J. L. O. (2006). Efeito de imagens estáticas com diferentes representações de movimento sobre a percepção subjetiva de tempo. Psicologia: Reflexão \& Crítica, 19, 217-224.

Nather, F. C., \& Bueno, J. L. O. (2011). Static images with different induced intensities of human body movements affect subjective time. Perceptual and Motor Skills, 113, 157-170.

Nather, F. C., \& Bueno, J. L. O. (2012). Timing perception in paintings and sculptures of Edgar Degas. Kronoscope: The Journal for the Study of Time, 12, 16-30.

Nather, F. C., Bueno, J. L. O., \& Bigand, E. (2013). Body movement induced by static images modulates eye movements and subjective time estimation. Psychology \& Neuroscience, 6, 261-270.

Nather, F. C., Bueno, J. L. O., Bigand, E., \& Droit-Volet, S. (2011). Time changes with the embodiment of another's body posture. PLoS One, 6, e19818.

Nicki, R. M., Lee, P. L., \& Moss, V. (1981). Ambiguity, cubist works of art, and preference. Acta Psychologica, 49, 27-41.

Osaka, N., Matsuyoshi, D., Ikeda, T., \& Osaka, M. (2010). Implied motion because of instability in Hokusai Manga activates the human motion-sensitive extrastriate visual cortex: an fMRI study of the impact of visual art. Neuroreport, 21, 264-267.

Proverbio, A. M., Riva, F., \& Zani, A. (2009). Observation of static pictures of dynamic actions enhances the activity of movementrelated brain areas. PLoS One, 4, e5389.
Russell, P. A. (2003). Effort after meaning and the hedonic value of paintings. British Journal of Psychology, 94, 99-110.

Silvia, P. J. (2005a). What is interesting? Exploring the appraisal structure of interest. Emotion, 5, 89-102.

Silvia, P. J. (2005b). Cognitive appraisals and interest in visual art: exploring an appraisal theory of aesthetic emotions. Empirical Studies of the Arts, 23, 119-133.

Smith, S. D., McIver, T. A., Di Nella, M. S., \& Crease, M. L. (2011). The effects of valence and arousal on the emotional modulation of time perception: evidence for multiple stages of processing. Emotion, 11, 1305-1313.

Stoyanova, Y., Yakimoff, N., Gourevich, A., \& Mitrani, L. (1987). In search of paintings influence on time judgment. Acta Neurobiologiae Experimentalis, 47, 103-109.

Strickland, C. (2004). The annotated Mona Lisa. Kansas City: John Boswell Management.

Thakral, P. P., Moo, L. R., \& Slotnick, S. D. (2012). A neural mechanism for aesthetic experience. Neuroreport, 23, 310-313.

Vartanian, O., \& Goel, V. (2004). Neuroanatomical correlates of aesthetic preference for paintings. Neuroreport, 15, 893-897.

Watts, F. N., \& Sharrock, R. (1984). Fear and time estimation. Perceptual and Motor Skills, 59, 597-598.

Wiesmann, M., \& Ishai, A. (2010). Training facilitates object recognition in cubist paintings. Frontiers in Human Neuroscience, 4, 11

Zakay, D. \& Block, R. A. (1996). The role of attention in time estimation processes. In: M.A. Pastor, \& J. Artieda (Eds.), Time, internal clocks and movement (pp. 143-164). Amsterdam: Elsevier. 